\title{
Determination of the obesity prevalence and its associated factors among community in selangor, Malaysia: An ordinal logistic regression approach
}

\author{
Noraznie Nordin ${ }^{1}$, Zalina Zahid ${ }^{2}$, Zaliha Ismail ${ }^{3}$, Siti Munira Yassin ${ }^{4}$, Hapizah Mohd Nawawi ${ }^{5}$, \\ Siti Aida Sheikh Hussin ${ }^{6}$ \\ ${ }^{1,2,5}$ Center of Statistical and Decision Sciences Studies, Faculty of Computer and Mathematical Sciences, \\ Universiti Teknologi MARA(UiTM), Malaysia \\ ${ }^{3,4}$ Faculty of Medicine, Universiti Teknologi MARA (UiTM), Malaysia \\ ${ }^{3,6}$ Institute for Pathology, Laboratory and Forensic Medicine (I-PPerForM), Universiti Teknologi MARA (UiTM), Malaysia
}

\begin{tabular}{l} 
Article Info \\
\hline Article history: \\
Received Oct 20, 2019 \\
Revised Dec 21, 2019 \\
Accepted Jan 5, 2020 \\
\hline
\end{tabular}

\section{Keywords:}

Biomarker

Cauchit link function

Obesity

Ordinal logistic regression

\begin{abstract}
Obesity is becoming an epidemic globally as it has been closely linked with a wide variety of chronic diseases. The identification of associated factors for obesity occurrences is still the main interest of many researchers. However, there has been extensive disagreement among researchers over possible factors associated with obesity which commonly involve the demographic factors, socioeconomic status (SES) and environmental factors. Biomarkers are also considered as important possible factors linked with the prevalence of obesity but investigations looking into their associations are still lacking. Therefore, it is important to examine factors that are associated with obesity using biomarkers and common factors to get detailed perspectives on obesity prevalence. The objectives of this study are to determine the prevalence of obesity and to examine the association between the common factors and biomarkers with obesity among community in Selangor, Malaysia. The results showed that the prevalence of obesity among participants was $49 \%(\mathrm{~N}=498)$ and Ordinal regression model with Cauchit built-in link function was the best fitted model to predict obesity. Meanwhile, three types of common factors (i.e. older age, being female and Malay ethnic) and one type of biomarker (i.e. high glucose level) were found to be significantly associated with obesity.
\end{abstract}

Copyright $\odot 2020$ Institute of Advanced Engineering and Science. All rights reserved.

\section{Corresponding Author:}

Zalina Zahid,

Center for Statistical and Decision Sciences Studies,

Faculty of Computer \& Mathematical Sciences,

Universiti Teknologi MARA, 40450 Shah Alam, Selangor, Malaysia.

Email: zalina@tmsk.uitm.edu.my

\section{INTRODUCTION}

Obesity known as a condition when an irregular or extreme fat accumulates in human body [1], is a recognized risk factor of chronic ill health. Despite enormous mounting recognition of the problem, the obesity rates are still increasing globally. In Malaysia alone, obesity prevalence has increased from $11.5 \%$ to $42.5 \%$ in 1975 and 2016 [2]. Commonly, the prevalence of obesity is found to be closely related to several chronic diseases such as hypertension, diabetes mellitus, heart diseases and certain types of cancer. However, obesity prevalence is also connected to individual's psychosocial well-being and nation's economic consequences [3, 4]. If the trend of high prevalence in obesity continues, future obesity-related healthcare costs may escalate and cause economic burden to the society [5]. 
Obesity can be considered as a preventable disease through the control and management of its potential associated factors. The identification of associated factors for obesity occurrences is still the main interest of many researchers. There has been extensive disagreement among researchers [6-9] over possible factors associated with obesity. So far, typical associated factors of obesity which have been investigated by previous researchers [10-12] include the demographic factors, socioeconomic status (SES) and environmental factors.

Previous studies [13, 14] have found obesity prevalence varies considerably by demographic characteristics involving age, gender, ethnicity or racial and marital status among adults as well as children. Meanwhile Socioeconomic status (SES), typically is reflected by income level, education level and types of occupation, were found to be significantly associated with obesity in many studies [15-17]. It is expected also that environmental factor which involves lifestyle behavior of individuals, specifically in terms of their diet and level of physical activities involvement to have associations with obesity. A few studies [18-20] have confirmed environmental factors were related with obesity prevalence.

Biomarkers are also considered as important possible factors linked with the prevalence of obesity as it reflects participant's health status or intervention results. The components under a biomarker, consisting of inflammatory biomarkers, glucose level, Adipokines and Lipid profile, are used as indicators to measure fat distribution [21]. Although, biomarkers are considered as important factors linked with the prevalence of obesity, investigations looking into various biomarkers associated with obesity is still lacking. Therefore, it is important to examine the factors that are associated with obesity using biomarkers and common factors to get detailed and accurate perspectives on obesity prevalence.

Most attractive methods for uncovering the associated factors in medical fields are machine learning algorithm [22-24]. Although machine learning algorithm has been applied successfully in medical fields, its advantage requires massive data which lead to long processing time. To overcome time consuming convergence problem, Ordinal logistic regression is proposed in this paper for its easy implementation and fast convergence rate in predicting obesity class with high degree of accuracy. This paper aims to determine the prevalence of obesity and examine the association between the common factors and biomarkers with obesity among community in Selangor, Malaysia using Ordinal Regression Approach.

\section{RESEARCH METHOD}

Figure 1 illustrates the research framework of this study. First stage involves data compilation from health records under Institute for Pathology, Laboratory and Forensic Medicine (I-PPerForM), Universiti Teknologi MARA (UiTM), Sungai Buloh, Selangor, Malaysia. The compiled data cover a total of 498 records of participants who had voluntarily attended community health screening programs conducted by a Clinical Training Centre of UiTM. Next stage proceeds to data selection involving compilation of data covering socio-demographic factors namely, age, gender, race, smoking status, family history of premature Coronary Artery Disease (CAD) and marital status. Meanwhile, data on four types of biomarkers namely, glucose level, lipid profile Adipokines and Inflammation were also compiled. These two groups of data were chosen to ensure the comprehensiveness of overall associated factors with obesity. Meanwhile, Body Mass Index of participants, which are classified into three groups (i.e. Underweight and Normal Weight, Overweight, Obese), was utilised as the potential indicator of obesity. Next, third stage involves data preprocessing, where missing data and outliers were examined. The total percentage of the missing data in this study was $9.1 \%$ and a number of outliers were observed through the box-plots. Overcoming these problems require Multiple Imputation to impute missing values and Winsorization technique to replace outliers with percentile values to ensure robust means in the data analysis. Further fourth and fifth stages focus on statistical analyses which were performed using Statistical Program for Social Science Software (SPSS). Initially, Chi-square test was conducted to detect the associations between categories of obesity and sociodemographic factors. Then, as part of validation of the Ordinal logistic regression, multicollinearity assumption was examined using variance inflation factor (VIF) and tolerance statistics. Next, statistical analysis involving Ordinal regression model was fitted to model the association between obesity response as dependent variable and potential associated factors as independent variables. Ordinal logistic regression is the appropriate model for the analysis tool as obesity response was recorded in terms of 3-level ordinal scale. Using this model, the log odds of a person being in one of the weight categories was modelled as a linear function of predictor variables. Specifically, three link functions, namely Complementary log-log, Probit and Cauchit were applied under the assumption the distribution of body mass index follows normal distribution with high probability of higher category. Then, the following stage is to determine the best Ordinal logistic regression model using two criteria i.e. minimum Akaike Information Criterion (AIC) value and higher value of Nagelkerke's R-square. Finally, the statistical analysis continued to identify significant factors (i.e. demographic factors and biomarkers) that were associated with increased odds of obesity. 


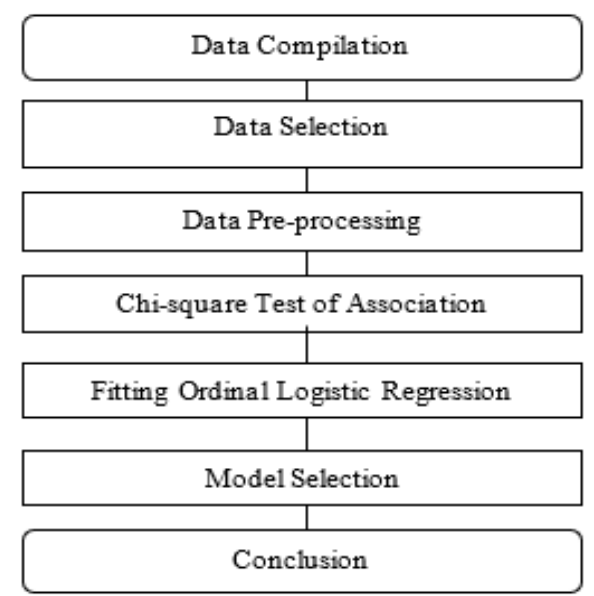

Figure 1. Research framework

\subsection{General linear model for ordinal data}

In this study, the proportional odds model or cumulative probabilities for $\mathrm{j}$ predictors with $\mathrm{j}-1=3$ $1=2$ levels of body mass index are given in (1). In ordinal logistic regression, the slope coefficient vectors are the same for each equation and only the intercepts are different.

$$
\mathbf{P}(\gamma \leq j)=\frac{\exp \left(\alpha_{j}\left(\beta_{1} \mathrm{X}_{i}+\ldots+\beta_{k} \mathrm{X}_{k}\right)\right)}{1+\exp \left(\alpha_{j}\left(\beta_{1} \mathrm{X}_{i}+\ldots+\beta_{k} \mathrm{X}_{k}\right)\right)} \text { for } \mathrm{j}=1,2
$$

where,

$\alpha_{j}=$ Called threshold

$\beta_{1}=$ Parameter

$\mathrm{X}_{i}=$ Factor of predictor

Each logit has its own $\alpha_{j}$ term but has the same coefficient $\beta$ which indicates that the impact of the independent variables is the same for different logit functions. The parameters are estimated using maximum likelihood estimation. In ordinal model, the logit expression is equal to the probability of being in or below a given group. By taking the logit transformations of both sides, the logits of cumulative probabilities are expressed in terms of (2).

$$
\operatorname{Logit}[P(\mathrm{Y} \leq j)]=\log \left[\frac{P(\mathrm{Y} \leq j)}{1-P(\mathrm{Y} \leq j)}\right]=\alpha_{j}-\left(\beta_{1} \mathrm{X}_{i}+\ldots+\beta_{k} \mathrm{X}_{k}\right) \text { for } \mathrm{j}=1
$$

Since $\mathrm{j}=2$ (Body mass index), the models used in this study are written as in (3) and (4).

$$
\operatorname{Logit}[P(\mathrm{Y} \leq 1)]=\log \left[\frac{P(\mathrm{Y}=1)}{P(\mathrm{Y}=2)+P(\mathrm{Y}=3)}\right]=
$$

logit for being in or below Underweight and Normal weight

$$
\operatorname{Logit}[P(\mathrm{Y} \leq 2)]=\log \left[\frac{P(\mathrm{Y}=1)+P(\mathrm{Y}=2)}{P(\mathrm{Y}=3)}\right]=
$$

logit for being in or below Overweight

\subsection{Logit model for ordinal responses}

Applying ordinal logistic regression for modelling obesity occurrences requires link functions to transform cumulative probabilities of obesity occurrences as part of model estimation. In this study, three types of link functions were considered as follows: 


\subsubsection{Complementary log-log}

Complementary log-log function denotes the inverse of the negative log-log function. It is appropriate when the probability of the higher category of an event is high. Mathematically, complementary $\log -\log$ can be expressed as:

$$
\begin{aligned}
& P(\mathrm{Y} \leq j)=\log (-\log (1-P(\mathrm{Y} \leq j)))=\alpha_{j}-\left(\beta_{1} \mathrm{X}_{i}+\ldots+\beta_{k} \mathrm{X}_{k}\right) \\
& \text { for } \mathrm{j}=1,2
\end{aligned}
$$

\subsubsection{Probit}

Probit function of the cumulative normal distribution, is represented by $\mathrm{Z}$ score:

$$
P\left(\mathrm{Y}_{i} \leq j\right)=\phi(X \beta+\varepsilon)=\phi^{-1}(P(Y \leq j))=X \beta+\varepsilon=\mathrm{Y}^{\prime} \text { for } \mathrm{j}=1,2
$$

\subsubsection{Cauchit}

Cauchit link function is useful under the presence of extreme values in the dataset. Mathematically, Cauchit can be described as:

$$
P\left(Y_{i} \leq j\right)=\tan ((P(\mathrm{Y} \leq j)-0.5)) \text { for } \mathrm{j}=1,2
$$

\section{RESULTS AND ANALYSIS}

\subsection{Descriptive statistics of obesity and its associated factors}

Altogether there were 498 community participants aging 30 to 65 years old. Table 1 shows that the overall prevalence of obesity and overweight were found to be $49 \%$ and $36 \%$ respectively. In obesity category, $87 \%$ of participants were male and $26.8 \%$ were females. Further analysis shows that more than half $(50.7 \%)$ of Malay were obese as compared to non-Malay who was only 33.3\% obese. Meanwhile, the highest

\begin{tabular}{|c|c|c|c|c|c|}
\hline \multirow[b]{2}{*}{ Variable } & \multirow[b]{2}{*}{$\begin{array}{l}\text { Description of } \\
\text { Variable }\end{array}$} & \multicolumn{3}{|c|}{ Body Mass Index, (n, \%) } & \multirow[b]{2}{*}{ Total (n) } \\
\hline & & $\begin{array}{l}\text { Underweight and } \\
\text { Normal Weight }\end{array}$ & Overweight & Obese & \\
\hline \multirow{2}{*}{ Gender } & Female & $67(21.3)$ & $163(51.9)$ & $84(26.8)$ & 314 \\
\hline & Male & $9(4.9)$ & $15(8.2)$ & $160(87.0)$ & 184 \\
\hline \multirow{2}{*}{ Race } & Malay & $60(13.3)$ & $162(36.0)$ & $228(50.7)$ & 450 \\
\hline & Non-Malay & $16(33.3)$ & $16(33.3)$ & $16(33.3)$ & 48 \\
\hline \multirow{2}{*}{ Smoking Status } & No & $62(16.4)$ & $161(42.5)$ & $156(41.2)$ & 379 \\
\hline & Yes & $14(11.8)$ & $17(14.3)$ & $88(73.9)$ & 119 \\
\hline \multirow{2}{*}{ Marital Status } & Single & $6(11.8)$ & $19(37.3)$ & $26(51.0)$ & 51 \\
\hline & Married & $70(15.7)$ & $159(35.6)$ & $218(48.8)$ & 447 \\
\hline Family History & No & $42(12.8)$ & $115(35.1)$ & $171(52.1)$ & 328 \\
\hline Premature of CAD & Yes & $34(20.0)$ & $63(37.1)$ & $73(42.9)$ & 170 \\
\hline Obesity Classification & $\begin{array}{l}\text { No. of } \\
\text { participations }\end{array}$ & $76(15)$ & $178(36)$ & $244(49)$ & 498 \\
\hline
\end{tabular}
proportion of smoker $(73.9 \%)$ falls in the category of obese participants. However, surprisingly $52.1 \%$ of obese participants had no family history of premature of coronary artery disease (CAD).

Table 1. Frequency distribution of patients based on demographic variables and body mass index

Table 2 shows the data description of biomarker variables. Overall, the mean values of Glucose level of participants is $6.081 \mathrm{mmol} / \mathrm{L}$. As for Lipid profile, the averages of HDL, LDL, TG and Tc are 1.34 $\mathrm{mmol} / \mathrm{L}, 3.624 \mathrm{mmol} / \mathrm{L}, 1.663 \mathrm{mmol} / \mathrm{L}$ and $5.762 \mathrm{mmol} / \mathrm{L}$ respectively. Meanwhile, the components under Adipokines i.e. Adiponectin, Resistin and Visfatin have mean values of $13683.27 \mathrm{ng} / \mathrm{mL}$ and $5.279 \mathrm{ng} / \mathrm{mL}$ respectively. Finally, the inflammation type which consists of HsCRP level, IL-6 level, PAI-1 level and TPA level were measured to have averages from $2.864 \mathrm{mg} / \mathrm{L}, 7.068 \mathrm{pg} / \mathrm{mL}, 63.28 \mathrm{ng} / \mathrm{mL}$ and $4.852 \mathrm{ng} / \mathrm{mL}$ respectively.

Table 3 presents the results of Chi-Square Test between obesity and categorical variables i.e. gender, smoking status, race, marital status and family history of premature coronary artery disease (CAD). The analyses revealed that, there was a significant association between obesity with gender, race and smoking status. It was also found that marital status was not significantly associated with obesity but family history of premature coronary artery disease (CAD) was marginally significant associated with obesity. 
Table 2. Data description of biomarker variables

\begin{tabular}{|c|c|c|c|c|c|}
\hline Type of Biomarkers & Variables & Mean & Median & Minimum & Maximum \\
\hline \multirow[t]{2}{*}{ Glucose Level } & Glucose $(\mathrm{mmol} / \mathrm{L})$ & 6.081 & 5.300 & 1.900 & 22.300 \\
\hline & $\mathrm{HDL}(\mathrm{mmol} / \mathrm{L})$ & 1.34 & 1.30 & 0.60 & 2.90 \\
\hline \multirow{4}{*}{ Lipid Profile } & $\mathrm{LDL}(\mathrm{mmol} / \mathrm{L})$ & 3.624 & 3.7 & 0 & 9 \\
\hline & $\mathrm{TG}(\mathrm{mmol} / \mathrm{L})$ & 1.663 & 1.4 & 0.4 & 9.3 \\
\hline & $\mathrm{TC}(\mathrm{mmol} / \mathrm{L})$ & 5.762 & 5.7 & 2.6 & 10.7 \\
\hline & Adiponectin (ng/mL) & 13683.27 & 10349.38 & 7.74 & 74634.87 \\
\hline \multirow[t]{2}{*}{ Adipokines } & Resistin (pg/ml) & 3628.5 & 2140.6 & 160.6 & 37552.0 \\
\hline & Visfatin (ng/mL) & 5.279 & 4.190 & 0 & 36.930 \\
\hline \multirow{4}{*}{ Inflammation } & hs-CRP (mg/L) & 2.864 & 2 & 0 & 9.9 \\
\hline & Il-6 (pg/mL) & 7.068 & 4.365 & 0.080 & 155.640 \\
\hline & PAI-1 (ng/mL) & 63.28 & 43.70 & 6.55 & 454.14 \\
\hline & $\mathrm{t}-\mathrm{PA}(\mathrm{ng} / \mathrm{mL})$ & 4.852 & 3.260 & 0.48 & 3.260 \\
\hline
\end{tabular}

Table 3. The association between obesity and independent variables

\begin{tabular}{|c|c|c|c|c|c|c|}
\hline \multirow[b]{2}{*}{ Variable } & & \multicolumn{3}{|c|}{ Body Mass Index, n (\%) } & \multirow{2}{*}{$\begin{array}{c}\text { Chi-squared } \\
\text { Value* }\end{array}$} & \multirow[b]{2}{*}{ P-Value } \\
\hline & & $\begin{array}{l}\text { Underweight and } \\
\text { Normal Weight }\end{array}$ & Overweight & Obese & & \\
\hline \multirow{2}{*}{ Gender } & Female & $67(21.3)$ & 163(51.9) & $84(26.8)$ & \multirow{2}{*}{168.541} & $<0.0001 *$ \\
\hline & Male & $9(4.9)$ & $15(8.2)$ & $160(87.0)$ & & \\
\hline \multirow{2}{*}{ Race } & Malay & $60(13.3)$ & $162(36.0)$ & $228(50.7)$ & \multirow{2}{*}{14.114} & $0.001 *$ \\
\hline & Non Malay & $16(33.3)$ & $16(33.3)$ & $16(33.3)$ & & \multirow{3}{*}{$<0.0001 *$} \\
\hline \multirow{2}{*}{ Smoking Status } & No & $62(16.4)$ & $161(42.5)$ & $156(41.2)$ & \multirow{2}{*}{41.266} & \\
\hline & Yes & $14(11.8)$ & $17(14.3)$ & $88(73.9)$ & & \\
\hline \multirow{2}{*}{ Marital Status } & Single & $6(11.5)$ & $19(37.3)$ & $26(51.0)$ & \multirow{2}{*}{0.537} & \multirow{2}{*}{0.764} \\
\hline & Married & $70(15.7)$ & $159(35.6)$ & $218(48.8)$ & & \\
\hline \multirow{2}{*}{$\begin{array}{l}\text { Family History of } \\
\text { Premature CAD }\end{array}$} & No & $42(12.8)$ & $115(35.1)$ & $171(52.1)$ & \multirow{2}{*}{5.855} & \multirow{2}{*}{0.054} \\
\hline & Yes & $34(20.0)$ & $63(37.1)$ & $73(42.9)$ & & \\
\hline
\end{tabular}

* Chi-squared Test is significant at 0.05 level (2-tailed)

\subsection{Ordinal logistic regression analysis}

Data pre-processing was executed to ensure missing data, outliers' existences and multicollinearity problems did not exist in the data set. Model evaluation involves comparisons of estimates, Nagelkerke $R^{2}$ and AIC values based on three models with different built-in link functions. Table 4 presents the coefficient estimates of associated obesity factors based on final ordered regression model with three built-in link functions. The results showed that out of 18 covariates, only 3 covariates (under Complementary log-log), 5 covariates (under Probit) and 4 covariates (under Cauchit) were significantly associated with obesity.

Table 4. Coefficient estimates of associated obesity factors based on ordinal regression model (Reduced Model)

\begin{tabular}{|c|c|c|c|c|c|c|}
\hline \multirow{3}{*}{ Variables } & \multicolumn{6}{|c|}{ Ordinal regression models } \\
\hline & \multicolumn{2}{|c|}{ Complementary log-log } & \multicolumn{2}{|c|}{ Probit } & \multicolumn{2}{|c|}{ Cauchit } \\
\hline & Estimate & SE & Estimate & SE & Estimate & SE \\
\hline $\begin{array}{c}\text { Body Mass Index } \\
\text { (Underweight and Normal Weight |Obese) }\end{array}$ & -2.840 & .485 & -2.074 & .412 & -3.098 & .872 \\
\hline Body Mass Index (Overweight | Obese) & -1.135 & .476 & -.671 & .406 & -.039 & .884 \\
\hline Glucose & $-.199 *$ & .049 & $-.217 *$ & .044 & $-.528 *$ & .099 \\
\hline High Sensitivity C-Reactive protein & - & - & $-.048 *$ & .022 & - & - \\
\hline Age & $.042 *$ & .008 & $.043 *$ & .007 & $.100^{*}$ & .018 \\
\hline Gender (Female vs. Male) & $-2.392 *$ & .217 & $-1.700 *$ & .144 & $-4.261 *$ & .519 \\
\hline Race (Malay vs. Non Malay) & - & - & - & - & $.872 *$ & .346 \\
\hline $\begin{array}{c}\text { Smoking Status } \\
\text { (Non-Smoker vs. Smoker) }\end{array}$ & - & - & $-.374 *$ & .151 & - & - \\
\hline
\end{tabular}

*Significant at $5 \%$ level of significance $(\mathrm{p}<.05) ; \mathrm{SE}=$ Standard error; ${ }^{-}$Not included in the model

Table 5 display the model performance measures based Nagelkerke $R^{2}$ and AIC values. Based on AIC values, it is revealed that the ordinal regression model with Cauchit built-link function was the best fit (769.077), followed by Complementary log-log built-in link function (812.7061) and the least fit model was Probit built-in function (780.5354). This is further supported by Nagelkerke $R^{2}$ values where Nagelkerke 
$R^{2}$ is the highest in ordinal regression model with Cauchit built-in link function $(0.456)$, followed by Probit built-in link function (0.431) and Complementary log-log built-in link function (0.416). Overall, Ordinal regression model with Cauchit built-in link function was found to be the best fit model to determine the associated factors of obesity.

Table 5. Nagelkerke $R^{2}$ and AIC values of the ordinal regression model with different built-in link functions

\begin{tabular}{ccc}
\hline Analysis (Link) & $R^{2}$ & AIC \\
\hline Ordinal Logistic Regression & 0.416 & 812.7061 \\
(Complementary log-log) & & \\
Ordinal Logistic Regression (Probit) & 0.431 & 780.5354 \\
Ordinal Logistic Regression (Cauchit) & 0.456 & 769.077 \\
\hline
\end{tabular}

Therefore, the final models are specified as below:

a) Cauchit $[\mathrm{P}(\mathrm{Y} \leq$ Underweight and Normal Weight $)]=-3.098-0.528($ Glucose level $)+0.100($ Age $)-$ 4.261(Female) + 0.872(Malay)

b) Cauchit $[\mathrm{P}(\mathrm{Y} \leq$ Overweight $)]=-0.039-0.528($ Glucose level $)+0.100($ Age $)-4.261($ Female $)+$ 0.872(Malay)

Best fitted model involving Ordinal logistic regression with Cauchit link function showed that only glucose level, age, being female and Malay participants were significantly associated with increased odds of obesity among community in Selangor. The implication is that older and Malay participants were more likely being the chance to be obese. This result is supported by [25], where their results also found elders are at more risk of many non-communicable diseases such as obesity. Furthermore, [25] also indicated that increases in prevalence of obesity were noticeably among both adults in many countries throughout the world. For example, in Great Britain, obesity cases almost tripled between 1980 and 2002 [25, 26]. The results also showed that Malay participants tend to be obese as compared to non-Malay. This finding is supported by another study [27] that showed positive association between race and obesity.

Meanwhile, it was found that high glucose level and female participants were less likely being the chance to be obese. It is common that participants who have high glucose level were less likely to have a chance of being classified as having obese [28] as the body fat prevented the glucose level to raise [29]. On the other hand, females were also found to be less likely to be obese as compared to male participants. This result is consistent with previous study [30] that reported females were at higher risk of obesity as compared to males due to low level of involvement in physical activity.

\section{CONCLUSION}

This study found that Ordinal regression model with Cauchit built-in link function is the best fitted model to determine the associated factors of obesity as compared to Probit built-in link function and Complementary log-log built-in link function Based on the evaluation results of the proposed model, Ordinal regression model with Cauchit built-in link function obtained the highest value of goodness of fit based on AIC value (769.007) and the largest value of explanatory power in terms of explaining data variations as represented by Nagelkerke $R^{2}(46 \%)$. Best fitted model involving the Ordinal logistic regression with Cauchit link function also showed that only glucose level, age, being female and Malay participants were significantly associated with obesity among community in Selangor. Obesity can be considered as a preventable disease through the control and management of its potential associated factors.

\section{ACKNOWLEDGEMENTS}

The authors would like to extend their gratitude and appreciation to Faculty of Computer and Mathematical Sciences, Universiti Teknologi MARA Shah Alam for the financial support to enable this paper to be published.

\section{REFERENCES}

[1] World Health Organization, "Noncommunicable diseases country profiles," 2011.

[2] World Health Organization, "Obesity and overweight," 2017.

[3] Finkelstein, E. A., et al., "Obesity and severe obesity forecasts through 2030," American Journal of Preventive Medicine, vol. 42, no. 6, pp. 563-570, 2012. 
[4] Dixon, J. B, "The effect of obesity on health outcomes," Molecular and Cellular Endocrinology, vol. 316, no. 2, pp. 104-108, 2010.

[5] Wang, Z. H, and Kihl-Selstam, E. J, "Ketoacidosis occurs in both Type 1 and Type 2 diabetes-a population based study from Northern Sweden," Diabetic Medicine, vol. 25, no. 7, pp. 867-870, 2008.

[6] A. Shuib., et al., "Two-stage LP diet model for Malay households," IEEE Colloquium on Humanities, Science and Engineering, pp. 562-567, 2011.

[7] Noor, N. L. M., et al., "Predicting obesity from grocery data: A conceptual process framework," International Conference on Information and Communication Technology for The Muslim World (ICT4M), pp. 286-291, 2016.

[8] Noor, N. L. M., et al., "Predictive analytics: The application of j48 algorithm on grocery data to predict obesity," IEEE Conference on Big Data and Analytics (ICBDA), pp. 1-6, 2018.

[9] Anuar, A. F, "Classification and prediction on scholl children for food intake attitude toward food and beverage adverstising on television: KFC as a case study," Degree Thesis, Universiti Teknologi MARA, 2017.

[10] Kamaruddin, A. A., et al., "Modelling of binary logistic regression for obesity among secondary students in a rural area of Kedah," AIP Conference Proceedings, vol. 1605, no. 1, pp. 856-861, 2014.

[11] Doku, D. T., "Designated by weights: Obesity among university employees," Obesity Medicine, vol. 5, pp. 11-15, 2017.

[12] Pan, C. C., et al., "Prevalence of overweight and obesity among students with intellectual disabilities in Taiwan: A secondary analysis," Research in Developmental Disabilities, vol. 53, pp. 305-313, 2016.

[13] Gallagher, D., et al., "How useful is body mass index for comparison of body fatness across age, sex, and ethnic groups?," Am J Epidemiol, 143, no. 3, pp. 228-239, 1996.

[14] Ogden, C. L., et al., "Prevalence of overweight and obesity in the United States, 1999-2004," JAMA, vol. 295, no. 13, pp. 1549-1555, 2006.

[15] Dinsa, G. D., et al., "Obesity and socioeconomic status in developing countries: A systematic review," Obes. Rev, vol. 13, no. 11, pp. 1067-1069, 2012.

[16] Jin, M. J., et al., "Prevalence of overweight and obesity and their associations with socioeconomic status in a rural Han Chinese adult population," PloS one, vol. 8, no. 11, 2013.

[17] Brennan, S. L., et al., "Socioeconomic status, obesity and lifestyle in men: The geelong osteoporosis study," $J$. Men's Health, vol. 7, no. 1, pp. 31-41, 2010.

[18] Kelishadi, R., et al., "Trend in the prevalence of obesity and overweight among Iranian children and adolescents: a systematic review and meta-analysis," Nutrition, vol. 30, no. 4, pp. 393-400, 2014.

[19] Ramezankhani, A., Dolati, M. R., and Khodakarim, "The study on relationship between education, employment and economic status of parents with overweight and obesity in students of primary schools in different parts of Tehran," Iran J Nutr Sci Food Technol, vol. 7, no. 5, pp. 197-205, 2012.

[20] Salehiniya, H., et al., "The prevalence of overweight and obesity in children under 5 years in Tehran, Iran, in 2012: A population-based study," Research in cardiovascular medicine, vol. 5, no. 1, 2016.

[21] Biomarkers Definitions Working Group. "Biomarkers and surrogate endpoints: Preferred definitions and conceptual framework," Clin Pharmacol Ther, vol. 69, no. 3, pp. 89-95, 2001.

[22] Wiharto,W., Esti, S., and Vicka, C., "The methods of duo output neural network ensemble for prediction of coronary heart disease," Indonesian Journal of Electrical Engineering and Informatics, vol. 7, no. 1, pp. 51-58, 2019.

[23] Lailil, M., Nurul, H., and Dimas, J. H., "Prediction of hypertension drug therapy response using K-NN imputation and SVM algorithm," Indonesian Journal of Electrical Engineering and Informatics, vol. 15, no. 1, pp. 460-467, 2019.

[24] Hari, S., et al., "Hypertension drug suiatability evaluation based on patient condition with improved profile matching," Indonesian Journal of Electrical Engineering and Informatics, vol. 11, no. 2, pp. 453-461, 2018.

[25] Saeed, K. M. I., "Prevalence and associated risk factors for obesity in Jalalabad city-Afghanistan," Alexandria Journal of Medicine, vol. 51, no. 4, pp. 347-352, 2015.

[26] Ogden, C. L., et al., "Prevalence of overweight and obesity in the United States, 1999-2004," JAMA, vol. 295 , no. 13, pp. 1549-1555, 2006.

[27] Wang, Y., and Beydoun, M. A., "The obesity epidemic in the United States e gender, age, socioeconomic, racial/ethnic, and geographic characteristics: A systematic review and meta-regression analysis," Epidemiol. Rev, vol. 29, no. 1, pp. 6-28, 2017.

[28] Wang, J., et al., "Association of lower body mass index with increased glycemic variability in patients with newly diagnosed type 2 diabetes: A cross- sectional study in China," Oncotarget, vol. 8, no. 42, 2017.

[29] Kresser, C., "Not all fat people get diabetes, and not all diabetics are fat," 2017.

[30] Saeed, K. M. I., "Prevalence and associated risk factors for obesity in Jalalabad city-Afghanistan," Alexandria Journal of Medicine, vol. 51, no. 4, pp. 347-352, 2015. 